\title{
II. Arbeitsaufnahme im Zeichen der latenten Krise (1925-1929)
}

Daß die offizielle Gründung der Deutschen Akademie im Mai 1925 nicht die erhoffte Resonanz in der deutschen Öffentlichkeit fand, war schon den Gründern bewußt. Haushofer sowie Senator Pechel kritisierten bereits unmittelbar nach dem feierlichen Gründungsakt die mangelhafte Öffentlichkeitsarbeit. Haushofer trat deshalb sowie wegen seiner vielfältigen anderen Verpflichtungen im Juli 1925 von seinem Posten als Präsident der praktischen Abteilung zurück. ${ }^{1}$ Der in Berlin sitzende Pechel sah zudem die Geschäftsstelle der Akademie in München als eine Art Sinekure für pensionierte bayerische Offiziere und Beamte wie Generalsekretär Frey, Geschäftsführer Heinrich Graf von Luxburg und den Nachfolger Haushofers, Staatssekretär Hans von Welser, die auf kulturpolitischem Gebiet gänzlich unerfahren waren. ${ }^{2}$ Frey schrieb im Oktober 1925 an Pfeilschifter, eine der Ursachen für die noch mangelnde Verankerung des Gedankens der Deutschen Akademie im deutschen Volk sei die fehlende Pressearbeit. Daher empfehle sich die Einstellung eines Pressereferenten. Für die Besetzung des Postens habe man auch schon einen geeigneten Kandidaten ausfindig gemacht, den bei den „Dresdener Nachrichten " tätigen Journalisten Franz Thierfelder. ${ }^{3}$

\section{Ein neuer Pressereferent: Franz Thierfelder}

Die eingeholten Informationen über den anvisierten neuen Pressereferenten ${ }^{4}$ klangen in der Tat vielversprechend:

„Nach vertraulicher Auskunft der beiden Bürgermeister von Dresden ist Thierfelder ein auBerordentlich begabter Mann von 30 Jahren. Er hat als primus omnium die Fürstenschule in Meißen absolviert, war vorbildlich als Freiwilliger und später Offizier im Feld, hat voll Volkswirtschaft und Philologie studiert, war schriftstellerisch auf dem Gebiet der Durchforschung und Pflege der deutschen Kultur tätig. Er hat auch über Pflege und Verbreitung deutscher Kultur im In- und Ausland eine Denkschrift verfaßt, die im Auswärtigen Amt Beachtung fand. Dr. Thierfelder gilt als Mann von größter Arbeitsfreudigkeit, größter Redegewandtheit, Zuverlässigkeit und vornehmer Gesinnung. Er soll sich an jeder Arbeitsstätte alsbald Achtung und Wertschätzung erworben haben." ${ }^{5}$

1 Vgl. Haushofers Schreiben an Rudolf Pechel vom 10.6. 1925, abgedruckt in: Jacobsen, Haushofer, Bd. 2, Dokument 28.

2 BAK N1160/I/142, Notizen Pechels für die Senatssitzung in Köln am 22. 10. 1926.

3 BAB R51/1, Schreiben Freys an Pfeilschifter, 18. 10. 1925.

4 Thierfelder war im übrigen bereits der zweite Kandidat für den Posten, da die Akademie im Sommer 1925 kurzzeitig einen Journalisten namens Beckmann angestellt hatte, der aber nach wenigen Wochen schon wieder entlassen worden war.

5 BHStA MA 100128, Schreiben Pfeilschifters an Ministerpräsident Held, 17. 9. 1925. 
Der Deutschen Akademie war offenbar sehr an der Verpflichtung Thierfelders gelegen, denn mit monatlich 600 RM Anfangsgehalt war die Akademie bereit, ihm weitaus mehr zu bezahlen als Generalsekretär Frey und Geschäftsführer Luxburg, die als Teilzeitkräfte nur $250 \mathrm{RM}$ erhielten. Zusätzlich kam die Akademie für Thierfelders Umzug auf und bot ihm eine mehr als einjährige Kündigungsfrist.

Thierfelder erfüllte die in ihn gesetzten hohen Erwartungen. Mit ihm, der im Februar 1926 von Dresden nach München übersiedelte, hatte die Deutsche Akademie eine Arbeitskraft gewonnen, die bald weit über die Pressearbeit hinaus entscheidende Impulse für ihre weitere Entwicklung geben sollte. Thierfelders neuer Arbeitgeber wurde sich dessen schnell bewußt. Schon nach einem Jahr, im März 1927, setzte sich Pfeilschifter unter Hinweis auf Thierfelders geistige Regsamkeit, Eigeninitiative, Kontaktfreudigkeit und schnelle Arbeitsweise für ein höheres $\mathrm{Ge}-$ halt des neuen Mitarbeiters ein, um ihn der Akademie zu erhalten. Auch von Müller, Pfeilschifters Nachfolger, schätzte Thierfelders Arbeit und befürwortete 1928 eine erneute Gehaltserhöhung: „Es ist mir außerordentlich daran gelegen, Herrn Dr. Thierfelder bei der Deutschen Akademie zu behalten. Er hat gute Ideen und ist selbständig, hat journalistische Begabung und er ist eine besonders wertvolle Arbeitskraft."6

Da Thierfelder für die Entwicklung der Deutschen Akademie wie auch für die Wiedergründung des Goethe-Instituts nach 1945 eine entscheidende Rolle spielte, und folglich breiten Raum in dieser Studie einnimmt, sei hier kurz sein Lebensweg rekapituliert. ${ }^{7}$ Franz Felix Reinhold Thierfelder wurde am 24. April 1896 in Deutschenbora in Sachsen als Sohn einer protestantischen Arztfamilie geboren, zu deren Vorfahren der sächsische Jurist, Philosoph und Wegbereiter der Aufklärung in Deutschland, Christian Thomasius, zählte. Nach dem Abitur an der renommierten Fürstenschule in Meißen meldete er sich 1914 als Kriegsfreiwilliger zur Infanterie. Ab 1916 bekleidete er den Rang eines Leutnants, im Juli 1918 geriet er schwerverwundet und hochdekoriert in Nordfrankreich in französische Kriegsgefangenschaft, aus der er im Oktober 1919 entlassen wurde. Nach Sachsen zurückgekehrt, studierte er in Leipzig Germanistik, nordische Sprachen, Zeitungsund Staatswissenschaften sowie Volkswirtschaft. Im Jahre 1921 nahm er als Freiwilliger an der Niederschlagung des kommunistischen Aufstandsversuches in Mitteldeutschland teil. Er schloß das Studium 1922 mit einer Promotion zu einem Thema der schwedischen Literatur des 16. und 17. Jahrhunderts und einer Promotion in Volkswirtschaft über den Bund der Landwirte in Sachsen ab. Anschließend arbeitete er als Journalist für die "Ostdeutsche Morgenpost" in Beuthen,

6 BAB R51/10144, Schreiben Pfeilschifters an den Finanzausschuß der Deutschen Akademie, 18. 3. 1927 sowie BHStA MA 100128, Schreiben von Müllers an den Finanzausschuß, 8. 11. 1928.

$7 \mathrm{Zu}$ Thierfelder vgl. auch Helms-Hardcastle, Franz Thierfelder. Die folgenden Angaben basieren aber vor allem auf zwei erhaltenen Lebensläufen von 1938 und von 1945 sowie dem Fragebogen zur Entnazifizierung von 1945, die alle nicht von Hardcastle benutzt worden sind. Das erste Dokument befindet sich im Bundesarchiv Berlin in den Unterlagen des Berlin Document Centers in der Personalakte Thierfelders der Reichskulturkammer, die anderen beiden Dokumente in der Personalakte Thierfelders im Bestand der Deutschen Akademie (R51/10116). 
dann für die „Kattowitzer Zeitung“, die „Goslarer Zeitung“ und schließlich als Leitartikler und Redakteur für die "Dresdener Nachrichten“. Er betätigte sich nebenbei als Übersetzer aus dem Schwedischen. ${ }^{8}$ Außerdem war er, folgt man seinem Lebenslauf von 1938, mehr oder weniger des Holländischen, Afrikaans, Englischen, Französischen, Bulgarischen und Griechischen mächtig. Zum Zeitpunkt der Arbeitsaufnahme bei der Deutschen Akademie war er verheiratet und hatte zwei Töchter, ein Sohn wurde 1933 geboren.

Obwohl nicht parteipolitisch aktiv, war er wie die meisten seiner Generation, seines sozialen Hintergrunds und Bildungswegs kein Freund der Weimarer Republik. Er war vielmehr ihren konservativ-revolutionären Gegnern zuzurechnen, die dem angeblich oberflächlichen Materialismus des westlichen Parlamentarismus einen nebulösen, autoritären, angeblich sittlich höherstehenden, im Volkstum verwurzelten, „organisch" gewachsenen statt "mechanisch" konstruierten Staat entgegenstellen wollten. ${ }^{9}$ So schrieb er beispielsweise ganz im Stile der rechten Kritiker der Republik 1930:

„Gerade heute wächst die Sehnsucht nach einer tieferen Sinngebung des Lebens, der Liberalismus englischer Färbung, der ungehemmte Kapitalismus und das Streben nach Weltherrschaft haben keine Harmonie in das Leben der Völker zueinander gebracht und der Materialismus aller Schattierungen ist wissenschaftlich tot und praktisch als schwerste Bedrohung der Menschheit erkannt worden. ${ }^{10}$

In seinem Fragebogen vom Juli 1945 zur Entnazifizierung führte er u.a. den zwei Jahre älteren Edgar Julius Jung, der seit der Veröffentlichung seines Buchs „Die Herrschaft der Minderwertigen" 1927 als eine der wichtigsten Vordenker der Konservativen Revolution in Deutschland galt, als Freund auf. Dieser lebte seit Mitte der zwanziger Jahre ebenfalls in München. " Thierfelder publizierte in entsprechenden, rechts angesiedelten Organen wie den "Süddeutschen Monatsheften“, der „Deutschen Rundschau“ und der DVP-nahen „Deutschen Allgemeinen Zeitung". Der Ruf der Deutschen Akademie, der ihr außerhalb Bayerns vorausging, nämlich eine nationalistische Einrichtung zu sein, die z.B. unter ihren Senatoren keinen Sozialdemokraten zählte, dürfte Thierfelder daher kaum gestört haben. Ihr anfänglicher Arbeitsschwerpunkt - die Förderung des Auslandsdeutschtums - war ebenfalls Anreiz für ihn, nach München überzusiedeln. So bekannte er

8 So übersetzte er den zweibändigen Roman von Mailo Talvio, Die Kraniche, Hamburg 1927.

9 Vgl. Kurt Sontheimer, Antidemokratisches Denken in der Weimarer Republik. Die Ideen des deutschen Nationalismus, München 41992; Stefan Breuer, Anatomie der Konservativen Revolution, Darmstadt 21995.

10 Franz Thierfelder, Geistige Grundlagen kultureller Auslandsarbeit, in: Süddeutsche Monatshefte, 28 (1930/31), S. 225-229, hier S. 228.

11 Allerdings schien Thierfelder 1945 die Erwähnung Jungs wohl nicht wegen dessen politischer Gesinnung opportun, sondern weil Jung, Ghostwriter der berühmten regimekritischen „Marburger Rede“ des konservativen Vizekanzlers Franz von Papen, bereits Ende Juni 1934 im Zuge des angeblichen „Röhmputsches“ von den Nationalsozialisten ermordet worden war. Zu Jung vgl. Bernhard Jenschke, Zur Kritik der konservativ-revolutionären Ideologie in der Weimarer Republik. Weltanschauung und Politik bei Edgar Julius Jung, München 1971 sowie Larry Eugene Jones, Edgar Julius Jung. The Conservative Revolution in Theory and Practise, in: Central European History, 21 (1988), S. 143-174. 
zwanzig Jahre später, seine damalige eigene Auffassung auf seine ganze Generation übertragend, daß die Jugend im Reich nach dem Ersten Weltkrieg den Gedanken, die geistige Verbundenheit aller Deutschen in der Welt zu fördern, als eine gerade ihr vorbehaltene nationale Mission aufgefaßt habe. Schließlich habe es kein anderes Volk gegeben, welches so weit über den Erdball zerstreut gewesen sei. ${ }^{12}$

\section{Finanzielle Probleme}

Auch der Arbeitsantritt Thierfelders in München änderte vorerst nichts an den gravierenden Startproblemen der Deutschen Akademie. Ursache für die Schwierigkeiten waren weniger in der mangelnden Öffentlichkeitsarbeit begründet als darin, daß für die Öffentlichkeit wie auch für potentielle Förderer in den Regierungsstellen und der Wirtschaft das Aufgabenprofil und damit die Existenzberechtigung der neuen Institution schlechthin zunächst schwer erkennbar blieb. Die Akademie sah sich in den ersten zwei Jahren ihrer Existenz hinsichtlich künftiger Arbeitsfelder mit den verschiedensten Vorstellungen ihrer Präsidenten sowie Anträgen und Gesuchen seitens ihrer Senatoren, aber auch anderer Personen und Institutionen im In- und Ausland konfrontiert. Für die erste Hauptversammlung, die im Oktober 1926 in Köln stattfand, sah der Katalog an Forderungen hinsichtlich anzugehender Projekte u. a. die Erstellung einer Schriftenreihe vor, die in kurzer, verständlicher Form wesentliche Aspekte der deutschen Kultur dem In- wie Ausland erklären sollte. Ferner führte er eine zu erstellende Statistik deutscher Kulturstützpunkte im Ausland auf sowie ein zu erarbeitendes Verzeichnis aller deutschen oder ehemals deutschen Ortsnamen jenseits der Reichsgrenzen. Auch die Forderung nach Überprüfung ausländischer Schulbücher auf möglichen deutschfeindlichen Inhalt durch die Akademie wurde erhoben. ${ }^{13}$ Daneben ergingen an die Deutsche Akademie Stipendienanträge auslandsdeutscher Studenten und von Ausländern sowie Förderungsanträge deutscher Kulturinstitutionen im Ausland. Weitere Vorschläge sahen die Arbeit der Deutschen Akademie zukünftig auf dem Gebiet der Sprachpflege, vor allem im Kampf gegen Fremdworte im deutschen Sprachschatz, wozu im Juni 1926 eine Vereinbarung mit dem Deutschen Sprachverein geschlossen wurde, ${ }^{14}$ und in der Beschäftigung mit der Frage, ob Antiqua oder Fraktur die der deutschen Sprache angemessene Schriftart sei. ${ }^{15}$ Zur Beantwortung dieser Frage setzte die Akademie einen speziellen Fachausschuß ein, der im Juni 1927 tagte, ohne eine endgültige Entscheidung zu treffen und vielmehr weitere Forschungen auf diesem Gebiet forderte. ${ }^{16}$ Ähnlich bunt

12 Franz Thierfelder, Volk - Nation - Staat, Aachen 1947, S. 15.

13 NStAOl Best. 271-14/679, Schreiben Onckens an die Mitglieder der wissenschaftlichen Sektionen der Deutschen Akademie, 9. 10.1926.

14 Abgedruckt in: MdDA, 1 (1925/26), S. $226 \mathrm{f}$.

15 NStAOl Best. 271-14/679, Aufzeichnung Onckens über die Anträge für die Hauptversammlung 1926, 9. 10. 1926.

16 Das gesamte Heft für September/Oktober 1927 der „Mitteilungen“ ist dieser Frage gewidmet. Die Akademie selbst verfolgte in der Schriftfrage im übrigen einen Schlingerkurs, der in gewisser Weise Ausdruck ihres unklaren auslandskulturpolitischen Profils war: Die 
waren die Vorschläge für die Jahresversammlung im Oktober 1927: So wurde ein Ausschuß gefordert, der die Rechtschreibung orientalischer und slawischer Eigennamen, die in die deutsche Sprache Eingang gefunden hatten, einheitlich regeln solle. Ein anderer Antrag forderte die Bereitstellung von Geldern zur Herstellung von Kunstdrucken deutscher Meisterwerke der Malerei, um den Kunstgeschmack in den deutschen Haushalten zu heben, der angeblich durch kitschige oder ausländische Reproduktionen gefährdet sei. ${ }^{17}$

Selten jedoch rang man sich in München zu einem eindeutigen „Nein“ hinsichtlich der an die Akademie in den ersten Jahren herangetragenen Vorschläge zur kulturpolitischen Arbeit gegenüber dem In- wie Ausland durch. Deutlich wurde aus diesen Anträgen erneut, daß viele Senatoren und Vertreter der Ortsgruppen das Wirkungsfeld der Akademie in der Anfangsphase hauptsächlich im Hinblick auf das eigene Volk sahen, das vor dem Eindringen fremder Kultureinflüsse geschützt und zugleich sich seiner Nationalkultur, die es zu vereinheitlichen galt, stärker bewußt werden müsse. Im übrigen ließ man sich zwar gerne zum Senator der Akademie ernennen oder in eine ihrer wissenschaftlichen Sektionen berufen, doch hielt sich das Engagement für die Arbeit der Akademie bei Senatoren ${ }^{18}$ wie Wissenschaftlern in den Sektionen in Grenzen: „Da saß man abends heiter und angeregt mit alten und neuen Freunden zusammen, da freute man sich an wohlgelungenen Festreden und an ausgezeichneten Vorträgen, da sah man große Leute aus aller Welt. Aber wo waren die Leistungen, die dieser Deutschen Akademie ihr Schwergewicht gaben. Und wo waren die notwendigen Mittel?" So lautete das rückblickende Resümee des Germanisten Friedrich von der Leyen, Mitglied der Sektion Deutsche Sprache, Literatur und Volks- und Altertumskunde und von 1937 bis 1939 Präsident der wissenschaftlichen Abteilung. ${ }^{19}$

$\mathrm{Daß}$ die in den Gremien herrschende Unklarheit über Aufgaben und weitere Entwicklung der Akademie potentielle Unterstützer abschreckte, merkte u.a. der Publizist Paul Rohrbach auf seinen Werbereisen für die Akademie durch das Reich. Er war seit 1927 Leiter der Praktischen Abteilung und damit auch für die Verbreitung der Vorstellungen und Ziele der Akademie in Deutschland und die

ersten Ausgaben der „Mitteilungen“ wurden in Antiqua gedruckt. 1931 stellte man sie dann auf Antrag von Senator Karl Christian von Loesch, dem Vorsitzenden des „Deutschen Schutzbundes“, auf Fraktur um, da dieser Schrifttyp die Verbreitung deutscher Kultur unter Ausländern nicht wesentlich behindere, aber angeblich ein wichtiger Beitrag zum Erhalt der völkischen Eigenart der Auslandsdeutschen darstelle. Dabei blieb es vorerst, da auch Thierfelder in den dreißiger Jahren argumentierte, eine von der internationalen Norm abweichende Schriftform sei, verglichen mit den anderen Problemen bei der Erlernung einer fremden Sprache, ein zu vernachlässigender Faktor, während zugleich die gotische Schrift mit deutscher Kultur quasi gleichgesetzt werde. Im Jahre 1942 schließlich stellte die Akademie im Sinne der nunmehr „ausländerfreundlichen“ Schriftpolitik des NS-Regimes die „Mitteilungen“ wie auch die Zeitschrift „Deutschunterricht im Ausland“ auf die lateinischen Lettern um (vgl. hierzu Kapitel 6).

17 BAK N1160/I/142, Tagesordnung für die Sitzung des Senates, 14. 10. 1927.

18 Bei der Hauptversammlung der Deutschen Akademie in Jena im Oktober 1929 waren beispielsweise nur 10 Senatoren anwesend.

19 Friedrich von der Leyen, Leben und Freiheit der Hochschule. Erinnerungen, Köln 1960, S. 225. 
Betreuung der Ortsgruppen verantwortlich. So schrieb er im November 1928 anläßlich einer Rundreise in Sachen Deutsche Akademie aus Bamberg an Oncken, es sei ein klarer Arbeitsplan für die künftige Tätigkeit der Akademie vonnöten.

„Ich mache auch jetzt in Nordbayern wieder die Erfahrung, daß alles Werben und Reden vergeblich ist, wenn man den Leuten nicht sagen kann: die und die einleuchtende, begreifliche und bestimmte Sache wollen wir machen, und zu der bitten wir Euch um Hilfe. Was in der Akademie bisher geleistet worden ist, das will ich in keiner Weise verkleinern. Es hat aber doch keine rechte Werbekraft für weitere Kreise offenbart und hat nicht den Namen der Akademie über einen engeren Kreis hinaus bekannt gemacht. “20

Dabei schien Akzeptanz und Kenntnis der Deutschen Akademie und damit der Spendenzufluß um so schwächer zu werden, je weiter man sich von München entfernte. Das Süd-Nord-Gefälle in der Förderung zeigte sich zum Beispiel bei der Aktivität der Ortsgruppen. Sie hatten nicht nur die Idee und Arbeit der Deutschen Akademie bis in die hintersten Winkel Deutschlands zu tragen und damit zur einer Konstituierung einer geeinten Kulturnation beizutragen. Sie sollten zugleich einen Großteil der Mitgliedsbeiträge und Spenden an die Münchener Zentrale abführen, damit diese, so der ursprüngliche Gedanke, bei ihrer Finanzierung frei von jedweder staatlichen oder parteipolitischen Einflußnahme bleibe. Gerade beim Konzept der Finanzierung mittels Ortsgruppen machte sich die Tatsache, daß die Deutsche Akademie auf Initiative Münchener Honoratioren und in München gegründet worden war, nachteilig bemerkbar. Die Ortsgruppen manch kleinerer bayerischer Städte, Ende der zwanziger Jahre waren es schließlich 39 im ganzen Reich, trugen mehr zum Budget der Akademie bei als diverse Großstädte außerhalb Bayerns. So überwies die Ortsgruppe Berlin zwischen 1925 und 1930 nur 7000 RM, Leipzig 4770 RM, Köln 1760 RM und Bremen gar nur 600 RM nach München. Aus München selbst hingegen flossen 34230 RM in die Kassen, aus Nürnberg 16000 RM, aus Fürth 11367 RM und selbst Kitzingen überrundete Berlin an Beiträgen mit 7175 RM. ${ }^{21}$ Die Ortsgruppe Berlin, unter dem Namen „Berliner Freunde der Deutschen Akademie“ firmierend, und potentiell die wichtigste, da für den Kontakt zu den Reichsstellen verantwortlich, wurde, ebenso wie die Breslauer Ortsgruppe, anstatt Gelder nach München zu überweisen, schließlich sogar für ihre Repräsentationsaufgaben von Zuwendungen aus München abhängig.22 Auf der Hauptversammlung der Deutschen Akademie im Oktober 1929 in Jena klagte denn auch Hauptgeschäftsführer Fehn über die oft anzutreffende Auffassung, daß die Deutsche Akademie eine bayerische, einseitig konfessionelle und nationalistisch eingestellte Organisation sei, was der reichsweiten Verbreitung ihrer Idee entgegenstehe. 23

Auch unabhängig vom Süd-Nord-Gefälle und dem unklaren Tätigkeitsprofil der Akademie erwies sich die Idee, die Akademie durch Ortsgruppen zu finanzie-

20 NStAOl Best. 271-14/469, Brief Rohrbachs an Oncken, 27. 11. 1928.

21 BHStA MA 100128, Aufstellung über die Finanzen der Deutschen Akademie 1929/30, ca. Herbst 1930.

22 Müller, Lebenserinnerungen, S. 242.

23 Bericht über die 4. Hauptversammlung der Deutschen Akademie in Jena (17./18. 10 . 1929), in: MdDA, 4 (1929), S. 297-322, hier S. 319. 
ren, schon nach einem Jahr als nicht tragfähig. Die latente Wirtschaftskrise, in der sich die Weimarer Republik selbst in ihrer Phase der relativen Stabilität befand, verhinderte ohnehin den Zufluß von Spendengeldern in ausreichendem Maße. Schatzmeister Wilhelm Kisskalt, hauptberuflich Generaldirektor der Münchener Rückversicherungsgesellschaft, machte deutlich, daß nur großzügige Spenden aus der Wirtschaft oder Unterstützung von staatlicher Seite die Arbeit und den Ausbau der Akademie langfristig sichern könnten. ${ }^{24}$ Der jährliche Etat der Akademie, der sich aus den Zuwendungen der Ortsgruppen, Spenden aus der Wirtschaft und den Erträgen des Stiftungsvermögens von etwa 140000 RM zusammensetzte, bewegte sich in den zwanziger Jahren zwischen 90000 und 100000 RM.

Die von Kisskalt bereits 1926 anvisierte Alternative der Subventionierung der Arbeit durch Stellen des Reiches hatte jedoch in den zwanziger Jahren wenig Aussicht auf Realisierung. Gerade die Fixierung der Akademie auf die Auslandsdeutschen als Adressaten kultureller Aktivitäten, für die Gründer im Kontext der zwanziger Jahre zunächst naheliegend, erwies sich als nachteilig für die junge Organisation. Auf diesem Gebiet gab es eher ein Zuviel denn ein Zuwenig an Institutionen im Reich. Hier traf die Deutsche Akademie vor allem auf den 1908 aus dem „Deutschen Schulverein“ hervorgegangenen VDA und das 1917 gegründete DAI in Stuttgart, das vor allem über die Auslandsdeutschen forschte. Da sich die kulturpolitisch eher unerfahrenen Gründer der Deutschen Akademie offenbar wenig um die Tätigkeit bereits bestehender Einrichtungen gekümmert hatten, eckten sie schon in der Gründungsphase nicht nur bei den etablierten Akademien an, sondern auch bei den sich mit dem Auslandsdeutschtum beschäftigenden Institutionen. Die Idee, im Rahmen der Überarbeitung der „Allgemeinen Deutschen Biographie" in ihrer Sektion Geschichte auch eine Biographie bedeutender Auslandsdeutscher herauszugeben, rief z. B. im November 1926 einen geharnischten Protest aus Stuttgart hervor. Nicht zu Unrecht schrieb der Präsident des DAI, Theodor Wanner:

„Ich muß es unter diesen Umständen sehr lebhaft bedauern, daß die Deutsche Akademie bei derlei Arbeiten sich sowenig in Fühlung mit bestehenden Auslandsdeutschtumsverbänden hält, daß sie von diesen Arbeiten nichts weiß [...] Ich würde es sehr bedauern, wenn bei der Weiterbefolgung solcher Pläne durch die Deutsche Akademie das Deutsche Auslandsinstitut gezwungen wäre festzustellen, daß die Deutsche Akademie Gedanken hegt, die andere vor ihr gehabt haben und Arbeiten anfängt, von deren Beginn andere Deutschtumsverbände bereits offiziell Mitteilung gemacht haben. ${ }^{25}$

Der Plan wurde folglich fallengelassen.

Diese Konkurrenz zu etablierteren Institutionen im bereits intensiv beackerten Feld der Förderung und Betreuung des Auslandsdeutschtums verhinderte die Anerkennung und Förderung durch das Auswärtige Amt, dem hierfür federführenden Reichsressort. In einer „Aufzeichnung über die Vereine zur Förderung des Deutschtums im Ausland“ des Auswärtigen Amts vom 25. Januar 1927 fanden

${ }^{24}$ Bericht des Schatzmeisters Kisskalt für die Hauptversammlung der Deutschen Akademie 1926, in: Jacobsen, Haushofer, Bd. 2, Dokument 34.

25 NStAOl Best. 271-14/629, Schreiben des Deutschen Auslandsinstituts an die Deutsche Akademie, 9. 11. 1926. 
beispielsweise unter den insgesamt 14 aufgeführten Einrichtungen zwar neben den großen Organisationen wie dem VDA, der „Deutschen Stiftung“ und dem „Deutschen Schutzbund“ auch kleinere Organisationen wie die „Sächsische Jungenschaft", die landwirtschaftliche Praktikanten nach Siebenbürgen vermittelte, und der „Ausschuß für Minderheitenrecht“ Erwähnung. Die Deutsche Akademie hingegen fehlte in der Liste. ${ }^{26}$ Die Vorsitzenden der „Berliner Freunde der Deutschen Akademie" hatten bei einer Besprechung mit dem Leiter der Kulturabteilung des Auswärtigen Amts im März 1927 „den Eindruck einer befremdenden kühlen Einstellung gegenüber der Deutschen Akademie“, da laut Auswärtigem Amt ihre Gründung geeignet sei, die „Kreise“ anderer Verbände zu stören. ${ }^{27}$

\section{Aktivitäten $1925-1929$}

Worin bestand also die Aktivität der Akademie in der Anfangszeit? Die vier Sektionen der wissenschaftlichen Abteilung hatten sich unmittelbar vor bzw. kurz nach der Gründungsversammlung vom Mai 1925 konstituiert. Im Zentrum ihrer Anstrengungen stand zunächst eine Reihe von Gesamtausgaben der Werke bedeutender Deutscher, von denen jedoch keine bis zur Auflösung der Akademie im Jahre 1945 vollendet wurde. So arbeitete man in der Sektion für Deutsche Kunst und Musik an einer Werksausgabe des Komponisten Carl-Maria von Weber, von der bis 1930 noch kein Band erschienen war. Das zweite, Anfang der dreißiger Jahre abgeschlossene Großprojekt war die fotographische Erfassung des Regensburger Doms. Die Sektion für Deutsche Sprache, Literatur und Volks- und Altertumskunde bereitete eine Werksausgabe Jean Pauls vor, von der bis 1930 sechs Bände erschienen. Ein weiteres Großvorhaben war die Aufnahme deutscher Volkslieder auf Schallplatten. Ferner arbeitete sie an einem althochdeutschen Wörterbuch. Die Sektion für Deutsche Geschichte unterstützte die vom DreiMasken-Verlag betreute Gesamtausgabe der Werke Rankes, deren erste fünf Bände bis 1930 erschienen. Außerdem beschäftigte sie sich in Zusammenarbeit mit der Historischen Kommission der Bayerischen Akademie der Wissenschaften - beide Gremien waren personell weitgehend identisch - mit der Überarbeitung der zwischen 1875 und 1912 in 56 Bänden erschienenen „Allgemeinen Deutschen Biographie“, welche nach Auflösung der Deutschen Akademie 1945 von der Bayerischen Akademie der Wissenschaften weitergeführt und seit 1953 als „Neue Deutsche Biographie“ veröffentlicht wird. In der Sektion für Deutsche Staatsund Wirtschaftskunde war das Renommierprojekt die Herausgabe der Schriften des Ökonomen Friedrich List, von der bis 1930 vier Bände erschienen.

Die Arbeit an all diesen Projekten ging nur schleppend voran. Die Deutsche Akademie selbst hatte keine besoldeten wissenschaftlichen Kräfte, sondern an Festangestellten in der zweiten Hälfte der zwanziger Jahre lediglich eine mit sie-

26 PA Bd. R60300.

27 BAB R8043/1329, Sitzung des Vorstandes der Berliner Freunde der Deutschen Akademie am 22. 6. 1927, Protokoll vom 27.6. 1927. 
ben Festangestellten besetzte Geschäftsstelle. ${ }^{28}$ Die Akademie war daher auf die Mitarbeit von Wissenschaftlern von außen angewiesen, z. B. der Bayerischen Akademie der Wissenschaften. Sowohl der Präsident der wissenschaftlichen Abteilung wie die Leiter der Sektionen hatten anfangs kein Vetorecht, so daß zunächst neben den Renommierprojekten recht wahllos die Publikation von Einzelstudien durch Druckkostenzuschüsse unterstützt wurde. Die Akademie konnte aus ihrem ohnehin bescheidenen Budget - für alle Sektionen standen in den ersten fünf Jahren jährlich etwa 20000 bis 30000 RM zur Verfügung - neben Druckkostenzuschüssen lediglich Zusammenkünfte fördern. Oncken sprach 1928 auf der Hauptversammlung von einem Teufelskreis, in dem sich die Deutsche Akademie befinde: Einerseits werde kritisiert, daß es noch nicht genügend sichtbare Beweise für ihre Tätigkeit gebe, andererseits fehle es an Mitteln, wissenschaftliche Projekte rasch zum Ende zu bringen. ${ }^{29}$ Ebenso fehlte offenbar das Interesse der Öffentlichkeit an den Projekten, denn es wurde über die geringe Zahl von Subskribenten für die Carl-Maria-von-Weber-Ausgabe geklagt und über die nur schleppenden $\mathrm{Be}-$ stellungen für die ersten Bände der Ranke-Ausgabe.

Oncken geriet zunehmend ins Kreuzfeuer der Kritik. Auf der Hauptversammlung in Jena 1929 mußte er sich dem Vorwurf stellen, die Arbeit der wissenschaftlichen Abteilung sei zu zersplittert, da sie zufälligen Anregungen einzelner Mitglieder der Sektionen überlassen bleibe. Er dürfte daher froh gewesen sein, daß er seinen Posten durch seine bereits 1928 erfolgte Berufung auf einen Lehrstuhl in Berlin ohnehin abzugeben hatte, da laut Satzung alle Präsidenten eine Professur in München innehaben mußten. Er rechtfertigte die begonnenen aufwendigen Werksausgaben bedeutender Deutscher damit, daß man nur so die Bezeichnung Akademie verdiene. Die Versammlung beschloß dennoch, eine Kommission einzusetzen, die der Zersplitterung der wissenschaftlichen Arbeit ein Ende setzen sollte, indem sie einen Leitfaden für den auserkorenen neuen Präsidenten Arnold Oskar Meyer, Onckens Lehrstuhlnachfolger in München, ausarbeiten wollte. ${ }^{30}$ Oncken nahm, obwohl ursprünglich eines der Gründungsmitglieder, nach seiner Berufung nach Berlin keinen Einfluß mehr auf die Deutsche Akademie.

Litt schon die Arbeit der wissenschaftlichen Abteilung an einem fehlenden Gesamtplan und klarer Schwerpunktsetzung in den ersten Jahren, so war das Profil der praktischen Abteilung noch unklarer, was nicht weiter verwunderlich war, da sie ja schon mit einer nebulösen Agenda gestartet war. Die Tatsache, daß sie zwischen 1925 und 1930 drei Präsidenten hatte, war der Entwicklung eines eigenen Profils sicherlich auch nicht gerade förderlich. Nachdem Haushofer schon im Juli 1925 aus Enttäuschung über den schlechten Start der Akademie das Handtuch geworfen hatte, präsidierte ihr für zwei Jahre Hans Freiherr von Welser, ohne nach-

28 Der Stellenplan sah einen Geschäftsführer - bis 1926 Graf Luxburg, anschließend Major a.D. Richard Fehn -, den Pressereferenten Thierfelder, sodann eine halbe Stelle für den Leiter der praktischen Abteilung Rohrbach und schließlich noch drei Sekretärinnen und einen Registrator vor.

29 Bericht über die 3. Hauptversammlung der Deutschen Akademie (18.-20. 10. 1928), in: MdDA, 3 (1928), S. 1063-1083, hier S. 1073.

$30 \mathrm{Vgl}$. Bericht über die 4. Hauptversammlung der Deutschen Akademie (17./18. 10. 1929), in: MdDA, 4 (1929), S. 297-322, hier S. 300-317. 
haltigen Eindruck zu hinterlassen. Er trat ab, als er Mitte 1927 zum Staatssekretär im bayerischen Handelsministerium ernannt wurde. Ihm folgte im September 1927 auf Vorschlag Onckens der Publizist Rohrbach. Rohrbach hatte schon in seinem 1912 veröffentlichten Bestseller „Der deutsche Gedanke in der Welt" für eine intensivere kulturelle Selbstdarstellung im Ausland anstelle einer bloßen militärisch abgestützten deutschen Machtpolitik plädiert. ${ }^{31}$ Rohrbach war wie Oncken in der zweiten Hälfte des Ersten Weltkriegs gegen die überambitionierten und unrealistischen Kriegsziele der Alldeutschen und der neugegründeten „Deutschen Vaterlandspartei“ eingetreten. Beide bildeten für einige Jahre den eher liberalen Flügel in der insgesamt nationalkonservativ ausgerichteten Akademie. Rohrbach war in den zwanziger Jahren einer der bekanntesten Publizisten Deutschlands und erschien somit eine für die Zukunft der Akademie vielversprechende Wahl, zumal der gebürtige Baltendeutsche auch schriftstellerisch für die Belange des Auslandsdeutschtums eingetreten war. So war das erste Werk, das mit Unterstützung der Deutschen Akademie überhaupt erschien, sein Werk „Deutschtum in Not" gewesen. ${ }^{32}$ Doch sein Engagement für die Akademie wurde u.a. dadurch gebremst, daß er persönliche Probleme mit von Müller hatte. Nicht zuletzt deshalb erhielt er anders als seine Vorgänger nicht den Titel „Präsident der praktischen Abteilung " mit der laut Arbeitsvertrag vereinbarten entsprechenden Gehaltserhöhung, sondern wurde lediglich als „geschäftsführendes Präsidialmitglied“ geführt. Im Gegensatz zu seinen ehrenamtlichen Vorgängern Haushofer und Welser erhielt er aber immerhin ein Jahresgehalt von $10000 \mathrm{RM}$ von der Akademie.

Seine Idee, die Mitteilungen der Deutschen Akademie, die in den zwanziger Jahren eine Auflagenhöhe von 5000 Stück hatten, mit seiner Zeitschrift „Der deutsche Gedanke" zu verschmelzen, fand ebenfalls nicht die Zustimmung der anderen führenden Akademiemitglieder. Rohrbachs Amtsführung geriet auch unter wachsende Kritik seitens des Geschäftsführers Fehn sowie Thierfelders. ${ }^{33}$ Ein gewisser Neid darüber, daß Rohrbach, der laut Vertrag nur die Hälfte seiner Arbeitszeit der Akademie zu widmen brauchte, aber genauso viel verdiente wie der Vollzeit arbeitende Pressereferent/Generalsekretär und der Geschäftsführer, mag dabei eine gewisse Rolle gespielt haben. Thierfelder und Fehn warfen Rohrbach jedenfalls zu große Reisetätigkeit in eigener Sache vor. ${ }^{34}$ Im September 1930

31 Vgl. Walter Mogk, Paul Rohrbach und das „Größere Deutschland“. Ethischer Imperialismus im wilhelminischen Zeitalter. Ein Beitrag zur Geschichte des Kulturprotestantismus, München 1972 sowie Horst Bieber, Paul Rohrbach, ein konservativer Publizist und Kritiker der Weimarer Republik, Berlin 1972.

32 Paul Rohrbach, Deutschtum in Not! Die Schicksale der Deutschen in Europa außerhalb des Reiches, Berlin u.a. 1926.

33 Es ist nicht mehr feststellbar, wann Thierfelder offiziell vom Pressereferenten zum Generalsekretär avancierte, vermutlich aber nicht vor Anfang 1929, obwohl der erste Generalsekretär Frey bereits im Mai 1926 ausgeschieden war. In einem Schreiben an Held, den Vorsitzenden des Finanzausschusses, bezeichnete Thierfelder sich noch im Oktober 1928 als „Referent“. In: BHStA Nachlaß Heinrich Held, Bd. 1110. Später jedoch stellte Thierfelder seine Karriere stets so dar, als sei er bereits 1926 von der Akademie als Generalsekretär eingestellt worden.

34 Kritik Fehns und Thierfelders in BAB R51/1 anläßlich einer Besprechung über die Finanzen der Akademie (11.4. 1930). Einige Dokumente zu Rohrbachs Problemen finden sich 
schied Rohrbach aus seinem Amt aus. „Die Tätigkeit an der Akademie ist für mich eine unerfreuliche Erinnerung geworden“, bekannte er denn auch zwei Jahrzehnte später in seinen Memoiren. ${ }^{35}$ Nach seinem Abgang gab es keinen Präsidenten für die praktische Abteilung mehr. Sie wurde vielmehr direkt dem Präsidenten der gesamten Akademie unterstellt, erhielt aber wesentliche Prägungen durch Thierfelder. Inwieweit neben der Kritik an der Amtsführung Onckens und Rohrbachs durch die anderen Mitglieder des Führungszirkels der Akademie auch politische Differenzen dazu geführt haben mögen, daß die beiden vergleichsweise politisch liberal eingestellten Präsidiumsmitglieder nach 1929/30 den Kontakt zur Akademie vollständig abbrachen, ist rückblickend nicht mehr zu klären, aber nicht auszuschließen.

Im ersten Jahr ihrer Existenz war die praktische Abteilung vor allem mit dem Aufbau und der Betreuung der Ortsgruppen betraut. Selbst die Frage, ob die praktische Abteilung sich mehr auf das Aus- oder Inland konzentrieren solle, war innerhalb der Akademie anfangs strittig. Auf der ersten Hauptversammlung der Akademie im Oktober 1926 sahen einige Akademieangehörige ihre Zukunft eher darin, Anfragen an die Akademie zu bearbeiten bzw. an die Ortsgruppen oder die wissenschaftliche Abteilung zu überweisen. ${ }^{36}$ Die praktische Abteilung war zunächst auch forschend tätig, indem sie z. B. an der Erstellung eines Verzeichnisses von Ortsnamen deutschen Ursprungs außerhalb der Reichsgrenzen arbeitete, von dem aber letztlich nur der erste Band über Elsaß-Lothringen erschien. ${ }^{37}$

Bei ihrer Auslandsarbeit blieb sie in den zwanziger Jahren gemäß der sich bereits bei der offiziellen Gründung im Mai 1925 abzeichnenden Schwerpunktsetzung vor allem auf die kulturelle Betreuung der Auslandsdeutschen fixiert. Aufgrund einer Vereinbarung mit dem VDA vom Oktober 1925 blieb der Münchener Institution auf diesem Gebiet aber nur noch die „Fürsorge für die höheren geistigen Bedürfnisse" übrig, was jede Befassung mit dem Auslandschulwesen und Jugendfragen ausschloß. ${ }^{38}$ Diese Fürsorge beschränkte sich auf die Belieferung wissenschaftlicher Bibliotheken der Auslandsdeutschen mit Literatur, die Unterstützung von Museen wie dem Brückenthalmuseum in Hermannstadt in Siebenbürgen, die Förderung von Veröffentlichungen auslandsdeutscher Einrichtungen wie dem Herderinstitut in Riga oder des Sudetendeutschen Instituts in Wien und die Aufrechterhaltung von Auslandstheatern wie dem Deutschen Schauspielhaus in Memel und dem Deutschen Theater in Riga. Vereinzelt wurden auch Stipendien an Auslandsdeutsche zum Studium im Reich vergeben.

auch in seinem Nachlaß in BAK N1408/124 sowie im Nachlaß Onckens in NStAOl Best. 271-14/469.

35 Paul Rohrbach, Um des Teufels Handschrift. Zwei Menschenalter erlebter Weltgeschichte, Hamburg 1953, S. 286 ff. (Zitat S. 287).

36 Abgedruckt in: MdDA, 1 (1925/26), S. 259-267.

37 Deutsch-fremdsprachiges Ortsnamensverzeichnis, hrsg. im Auftrage der praktischen Abteilung der Deutschen Akademie, bearbeitet von Otto Kredel und Franz Thierfelder, Berlin 1931.

38 So die Bezeichnung in einem „Überblick über die gegenwärtige Tätigkeit der Deutschen Akademie" aus dem Jahre 1930, in: BHStA MK 40444. Die Vereinbarung ist abgedruckt in: MdDA, 1 (1925/26), S. $126 \mathrm{f}$. 
Die ersten Jahre des 1925 mit großem Anspruch offiziell lancierten kulturpolitischen Unternehmens „Deutsche Akademie“ waren also äußerst schwierig. Die schon in der Gründungsphase der Deutschen Akademie von diversen Seiten vorhandenen Widerstände und Vorbehalte gegen den Namen und die anvisierten Tätigkeitsfelder der entstehenden Institution, aber auch gegen die Tatsache, daß die neue Einrichtung ausgerechnet einer bayerischen Initiative entsprang, ließen nicht nach, im Gegenteil. Sie führten dazu, daß die Neugründung sich praktisch von Anbeginn an in einer permanenten finanziellen Krise befand, die nicht zuletzt aus dem für potentielle Förderer unklaren Arbeitsprofil der Akademie resultierte. Die schlecht abgegrenzte Aufgabenstellung wiederum war Resultat mangelnder Führung und kulturpolitischer Erfahrung der Gründer der Akademie. Ein weiterer Grund hierfür war die eine Entscheidungsfindung wie Finanzierung erschwerende Struktur, in der Präsidium, Geschäftsstelle, Senat und Ortsgruppen, die zwischen den Jahresversammlungen im sogenannten Kleinen Rat ${ }^{39}$ über den Kurs der Akademie berieten, in Einklang gebracht werden mußten. Personelle Rivalitäten im Führungszirkel zwischen Rohrbach und Oncken einerseits und von Müller, Thierfelder und Fehn andererseits taten ein übriges. Die Deutsche Akademie sah anläßlich ihres zehnjähriges Bestehens 1935 folglich ihre Anfänge durchaus selbstkritisch und treffend:

„Wir müssen heute zugeben, daß es der neuen Gründung an einem leicht verständlichen und kurzgefaßten Arbeitsplane gefehlt hat, mit dem man verwandte Vereinigungen von der Notwendigkeit einer Deutschen Akademie überzeugen konnte und die Öffentlichkeit von der Wichtigkeit ihrer Aufgaben zu erfüllen vermochte. [...] So war der Hauptvorwurf der ersten Jahre, die Deutsche Akademie komme zu spät, die Welt sei bereits verteilt, und diejenigen, die von Natur aus hätten Bundesgenossen sein sollen, witterten unerwünschten Wettbewerb und verhielten sich kühl." 40

39 In diesem saßen die Präsidenten, Schatzmeister, Geschäftsführer, Generalsekretär sowie Vertreter des Senats, der bayerische Ministerpräsident und ein Vertreter der Stadt München. Der in der Satzung vorgesehene sogenannte Große Rat war die Ergänzung des Gremiums um Vertreter der Ortsgruppen, doch tagte letzterer wegen der geringen Rolle, welche die Ortsgruppen letztlich spielten, kein einziges Mal.

40 Zehn Jahre Deutsche Akademie 1925-1935, in: MdDA, 10 (1935), S. 173-198, hier S. 178. 